\title{
A Study of Comparison between DJ Stenting and Silodosin after Ureteroscopic Lithotripsy for Mid and Lower Ureteric Calculus
}

\author{
Narendra Pai ${ }^{1}$, Rajeev Thekke Puthalath ${ }^{2}$, Suraj Hegde ${ }^{3}$, Amit Kumar ${ }^{4}$, Gaurav Kalra ${ }^{5}$ \\ ${ }^{1}$ Department of Urology, K. S. Hegde Medical Academy, Mangalore, Karnataka, India. ${ }^{2}$ Department of Urology, \\ KSHEMA, Mangalore, Karnataka, India. ${ }^{3}$ Department of Urology, KSHEMA, Mangalore, Karnataka, India. ${ }^{4}$ Department \\ of Urology, KSHEMA, Mangalore, Karnataka, India. ${ }^{5}$ Department of Urology, KSHEMA, Mangalore, Karnataka, India.
}

\section{ABSTRACT}

\section{BACKGROUND}

Ureteric calculi are known to affect approximately $10-15 \%$ of the overall population. We wanted to determine as to whether silodosin can be used instead of DJ stenting in patients with uncomplicated ureteroscopic lithotripsy.

\section{METHODS}

We selected 60 patients who underwent ureteroscopic lithotripsy (URSL) in the study group. They were divided into 'stented group' and 'non-stented group on silodosin'. Patients with stone of 5 to $18 \mathrm{~mm}$ size with no intraoperative mucosal injury and no stricture were included in the study. All patients underwent surgery using an 8/9.8 Fr rigid ureteroscope, without ureteral dilation, with lithotripsy using an electro-hydraulic lithotripter, without extraction. A 4.5 Fr Double J stent was placed in the first group for three to four weeks. The patients underwent urine routine examination, plain x-ray $\mathrm{KUB}$, and ultrasound abdomen before and after lithotripsy. Lower urinary tract symptoms and pain scores were recorded on 3, 7 and 15 days postoperatively. We compared mean operative time, emergency visits, rehospitalisation rates, and residual fragments between each group.

\section{RESULTS}

Out of 60 patients, $53.3 \%$ of patients had lower ureteric calculus, $30 \%$ had mid ureteric, and $16.7 \%$ had calculus at vesicoureteric junction. The mean calculus size on the left side was $10.23 \mathrm{~mm}$, and on the right side was $10.33 \mathrm{~mm}$. The mean intraoperative time was 33.23 minutes in stented and 29.9 minutes in the silodosin group ( $\mathrm{p}<0.003)$. Patients underwent assessment for flank pain, fever, and LUTS on postoperative days (POD) 3,7 and 15 . There was no statistically significant difference between the two groups though patients with DJ stent had more symptoms on inquiry. A total of 5 patients in the treatment groups were rehospitalised, two patients (6.7\%) in the stented group, and three patients $(10 \%)$ in the silodosin group, out of which three patients (5\%) required a secondary procedure which was not statistically significant. Three subjects treated with silodosin and 12 with the DJ stent in situ had residual fragments on POD 21 which was statistically significant ( $\mathrm{p}$ 0.27).

\section{CONCLUSIONS}

Patients with uncomplicated URSL have similar recovery of renal function when treated with silodosin as compared to the placement of DJ stent. Treatment without stent with silodosin also has less irritative LUTS. We conclude that silodosin can be an alternative to DJ stent after uncomplicated ureteroscopic electrohydraulic lithotripsy, thereby reducing operative time and patient morbidity.

\section{KEYWORDS}

Lithotripsy, Silodosin, DJ Stent
Corresponding Author:

Suraj Hegde,

Associate Professor,

Department of Urology,

K. S. Hegde Medical Academy,

Mangalore-575018, Karnataka, India.

E-mail: hegdesurajdr@gmail.com

\section{DOI: $10.14260 / j e m d s / 2020 / 384$}

Financial or Other Competing Interests: None.

How to Cite This Article:

Pai N, Puthalath RT, Hegde S, et al. A study of comparision between DJ stenting and silodosin after ureteroscopic lithotripsy for mid and lower ureteric calculus. J. Evolution Med. Dent. Sci. 2020;9(23): 1747-1751, DOI: 10.14260/jemds/2020/384

Submission 08-01-2020, Peer Review 12-05-2020, Acceptance 18-05-2020, Published 08-06-2020.

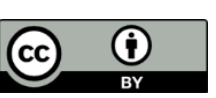




\section{BACKGROUND}

Ureteric calculi are known to affect approximately $10-15 \%$ of the overall population. The incidence of urolithiasis is rising over time.[1,2] Most of these stones are known to pass spontaneously with or without expulsive medical therapy. The expulsion of calculus depends on the following factors, the calculus size, and the location in the ureter. The calculi expulsion rates change depending on these factors, with the expulsion rate ranging from $40 \%$ to $98 \%$ for calculi less than $5 \mathrm{~mm} \cdot[3,4,5,6]$ The calculi, which measures $6 \mathrm{~mm}$ or more have a spontaneous expulsion rate ranging between $35 \%$ to $50 \%$.

The contraction of ureteral smooth muscles causes ureteric colic in the presence of stone due to the presence of $\alpha$-adrenergic receptors in them.[7,8,9] The reactive inflammation, which is caused by the calculus irritating the ureteric mucosa, produces mucosal oedema, thereby increasing stasis leading to obstruction. ${ }^{[7,8,9]}$ The density of these receptors is highest in the lower part of the ureter compared to the upper ureter.[9] The use of an $\alpha$-blocker dilates the ureter due to muscle relaxation while maintaining tonic propulsive contractions. So, use of $\alpha$-blocker results in the reduction in the intra-ureteric pressure facilitating in urinary transport.[7,8,9] URSL is a minimally invasive and effective procedure in the treatment of ureteric calculus. It is a preferred modality for managing ureteric calculi. The stonefree rate following ureteroscopy is higher compared to other treatment modalities for ureteric calculi. The availability of smaller scopes and advances in intracorporeal lithotripsy have resulted in the higher and successful treatment of ureteral calculi in recent years.

Silodosin is a selective $\alpha-1 \mathrm{~A}$ receptor antagonist. It is used in the management of benign prostatic hyperplasia. It has documented use in the therapy of ureteric calculus.

The treatment of ureteric calculi is tailored after discussion with the patient based on the following factors: success rates of individual treatment options, adjunctive procedures needed, and morbidity related to treatment. Both patient factors (body habitus, coagulation status, medical comorbidities) and stone factors (location, burden, composition) will be considered when selecting the optimal treatment for ureteric calculi. Use of Double J stent after ureteroscopy helps in the passage of residual fragments and prevents pain caused due to mucosal oedema and obstruction. However, many patients complain of stentrelated discomfort in the postoperative period. There is an additional need for stent removal, which is another surgical procedure adding to the cost of treatment. So, in our study, we want to know if silodosin can be an alternative to stenting after ureteroscopic lithotripsy. The use of silodosin can eliminate stent-related morbidity and the need for the second procedure.

\section{METHODS}

The study done was a Randomized controlled trial study. Sixty patients were included in the study and randomly assigned to two groups. The study group included patients with mid- or lower-ureteric calculus of size between 5-18 mm and posted for URSL. Exclusion criteria included patients with incomplete stone fragmentation needing secondary procedure, patients with ureteric stricture, those who underwent previous ureteric stenting, and those patients with signs of acute pyelonephritis or sepsis. Written informed consent was taken from all patients. After applying the inclusion and exclusion criteria, all eligible patients underwent further investigations like complete hemogram, Blood sugar assay, Renal function test, Urine routine and microscopy, Urine culture and sensitivity, USG Abdomen and pelvis, X-Ray KUB and NCCT as and when required. If calculus is radio-opaque, then X-Ray KUB was repeated on the morning of surgery to confirm its location before surgery.

A cystoscopy was done to place a safety guidewire of $0.025 / 0.032$ inch in the ureter under fluoroscopic guidance. We performed ureteroscopy using 8/9.8Fr semirigid URS. Calculus was fragmented completely using pneumatic lithotripter with a $1 \mathrm{~mm}$ probe till the fragments formed were smaller than the tip of the probe. The calculi fragments were not retrieved. After the complete fragmentation of the calculus, patients were divided into two groups randomly. In the first group, a $4.5 \mathrm{Fr}$ DJ stent was inserted, to be removed after three weeks. In the second group, patients were given one capsule of Silodosin $8 \mathrm{mg}$ in the night for three weeks.

Patients were discharged on postoperative day one if symptomatically better and followed up on postoperative days 3,7 , and 15 for symptomatic assessment. They further had to report if they had a fever, abdominal pain, or haematuria. Operative time, hospitalization time, pain score, LUTS, stone-free rate, rehospitalization, need for a second procedure were assessed in each patient. Ultrasound abdomen and pelvis and X-Ray was done on the 21st postoperative day to rule out any residual stones in the ureter.

\section{Sample Size}

Our sample size consisted of 60 subjects.

\section{Method of Allocation}

Random allocation of patients done to one of two treatment groups using a random number table envelope method SPSS version 16 was used for statistical analysis. For comparison between quantitative variables, the Student t-test was used. Qualitative variables were compared using the ChiSquare test.

\section{RESULTS}

A total of 60 patients were included in the study, $27 \%$ were female, and $73 \%$ were male patients. In our series, 26-35 years (22 patients) formed the predominant age group, followed by $36-45$ years (13 patients). $20 \%$ were in the age group of $46-55$ years; $16.7 \%$ of the patients belonged to 16 25 years of age. $5 \%$ of patients were in the age group of 5665 years. The mean age in the DJ stent group was 35.43 years, and in silodosin group was 37.57 years.

Among them, 29 patients (48.3\%) had right ureteric calculus, and 31 patients $(51.7 \%)$ had left ureteric calculus. The mean left-sided calculus size was $10.23 \mathrm{~mm}$, and the 
right-sided calculus was $10.33 \mathrm{~mm}$. There was no significant difference found between the mean size of calculus and the side. Thirty-two patients (53.3\%) had lower ureteric calculus, 18 patients $(30 \%)$ had mid ureteric calculus, and ten patients $(16.7 \%)$ had VUJ calculus. In our study, most of the patients, $41(68.33 \%)$, had radio-opaque calculus. Operative time included time from ureteroscope insertion until the removal of the endoscope. The mean operative time in the DJ stent group was 33.23 minutes, and in silodosin group was 29.90 minutes. There was a significant difference found in the operative time between the two groups. $(\mathrm{p}<0.003)$ (Graph 1$)$. The mean hospital stay in the DJ stent group was 2.60 days, whereas, in the silodosin group, it was 1.83 days (Table 1). There was a significant difference ( $p$ value $<0.009$ ) found between mean hospital stay among different treatment groups. In this study, a total of 5 patients in the treatment groups were re hospitalised. 2 patients $(6.7 \%)$ with a stent and three patients $(10 \%)$ on silodosin. However, the $p$ value was 0.640 . There was no significant difference found between Immediate/ Emergency Visit between two groups (Graph 2)

On day 3 of follow up, $43.3 \%$ of the DJ Stent group and $53.3 \%$ of the silodosin group were asymptomatic. $36.7 \%$ of patients in the DJ Stent group had only flank pain as compared to $40 \%$ of patients in the silodosin group. On comparing flank Pain \& LUTS, a majority $(13.3 \%)$ were in the DJ stent group, whereas only $3.3 \%$ of patients in the silodosin group had similar complaints. Finally, $6.7 \%$ of patients in the DJ Stent group complained of flank pain and haematuria, and only $3.3 \%$ in the silodosin group had such complaints. On statistical analysis, the $\mathrm{p}$ value was 0.377 . There was no significant difference found between treatment modalities concerning clinical features on day 3.

On day 7 of follow up, $63.3 \%$ patients were asymptomatic out of which $50 \%$ were in DJ stent group, and $76.7 \%$ were in silodosin group. $25 \%$ patients complained of flank pain, in which $30 \%$ were in the DJ Stent group, and 20\% were in the silodosin group. On comparing flank Pain \& LUTS, 3.3\% of patients were in the DJ stent group, whereas no patients in the silodosin group had similar complaints. $13.3 \%$ in the DJ stent group had flank pain \& haematuria, and only 3.3\% in the silodosin group had similar complaints. Finally, on comparing LUTS, $3.3 \%$ of patients in the DJ Stent group had LUTS, whereas none in the silodosin group complained of LUTS. On statistical analysis, the $\mathrm{p}$ value was 0.156 . clinical features on day 7 were similar in both groups, with no statistical differences.

On day 15 of follow up, $86.7 \%$ of patients in the silodosin group remained asymptomatic as compared to $63.3 \%$ of patients in the DJ stent group. Flank pain was present in $20.0 \%$ and $6.7 \%$ of patients with DJ stent and silodosin group, respectively. $3.3 \%$ of patients in the silodosin group had fever associated with flank pain, but none in the DJ stent group had fever. Haematuria associated with flank pain was present in $3.3 \%$ in each group. No patients in the silodosin treated group had LUTS associated with flank pain, whereas $10 \%$ of patients in the DJ stent group had LUTS with flank pain. Finally, LUTS alone was present in $3.3 \%$ patients with the stent, whereas no patient complained of LUTS in the silodosin group on the 15th day of follow up. However, on statistical analysis, $p$ value was 0.246 , and there was no significant difference statistically in clinical features on the 15th day between the treatment group.

In our study, we performed a USG abdomen and pelvis/ KUB x-ray on the 21st postoperative day to look for any residual fragments and to know the stone clearance rates. Twelve patients $(40 \%)$ in the DJ Stent group had residual fragments, whereas only three patients $(10 \%)$ in the silodosin group had residual fragments. On statistical analysis, the $\mathrm{p}$ value was 0.027 , which was indicative of a significant statistical difference between residual Fragments in the different treatment groups. (Graph 3)

In our study, a total of 3 patients required secondary procedure, one patient (3.3\%) in the DJ Stenting group, and two patients $(6.7 \%)$ in the silodosin group. On statistical analysis, the $\mathrm{p}$ value was 0.839 there was no significant difference found between Secondary procedure in treatment groups. (Table 2, Graph 4)
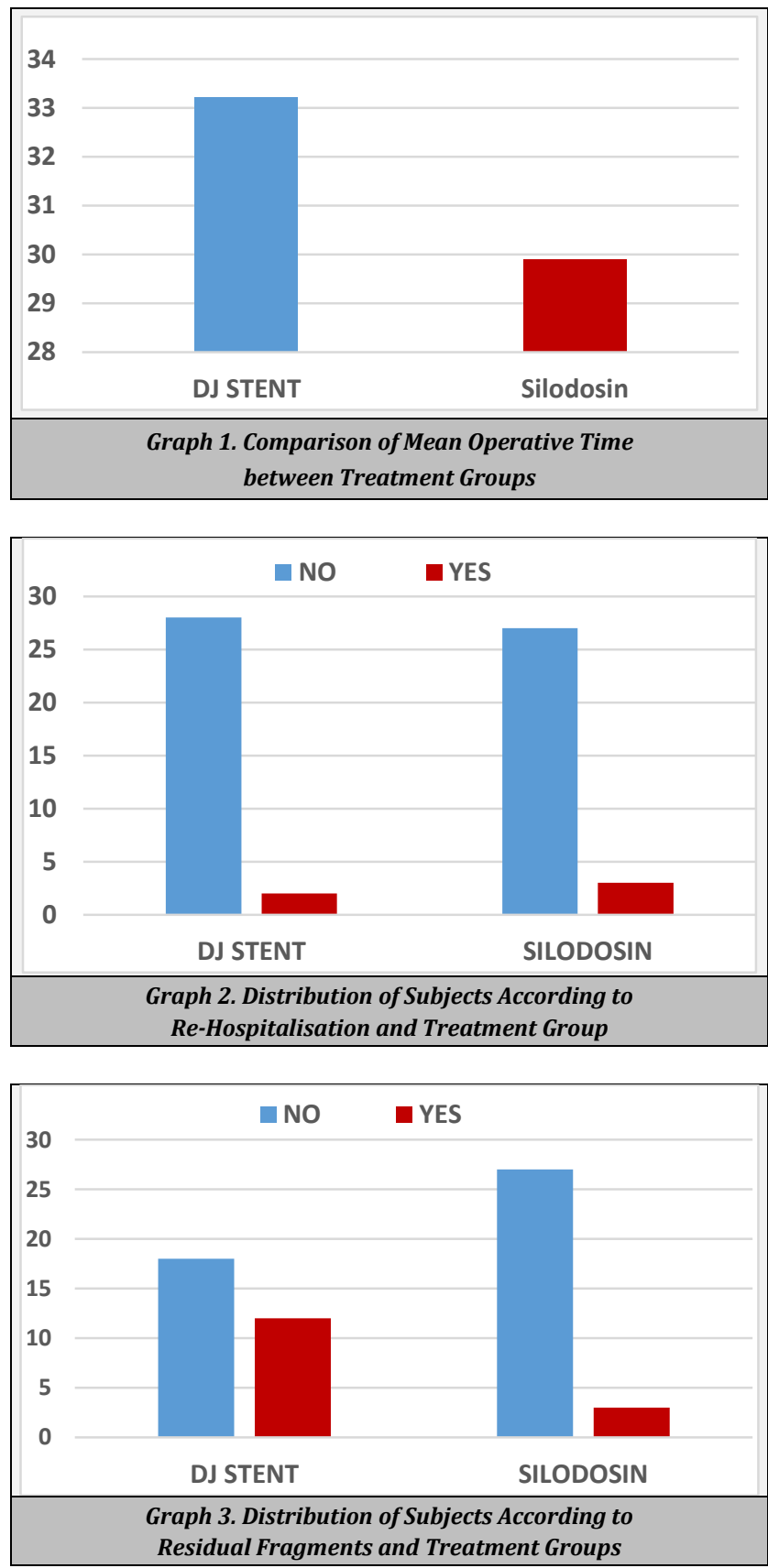


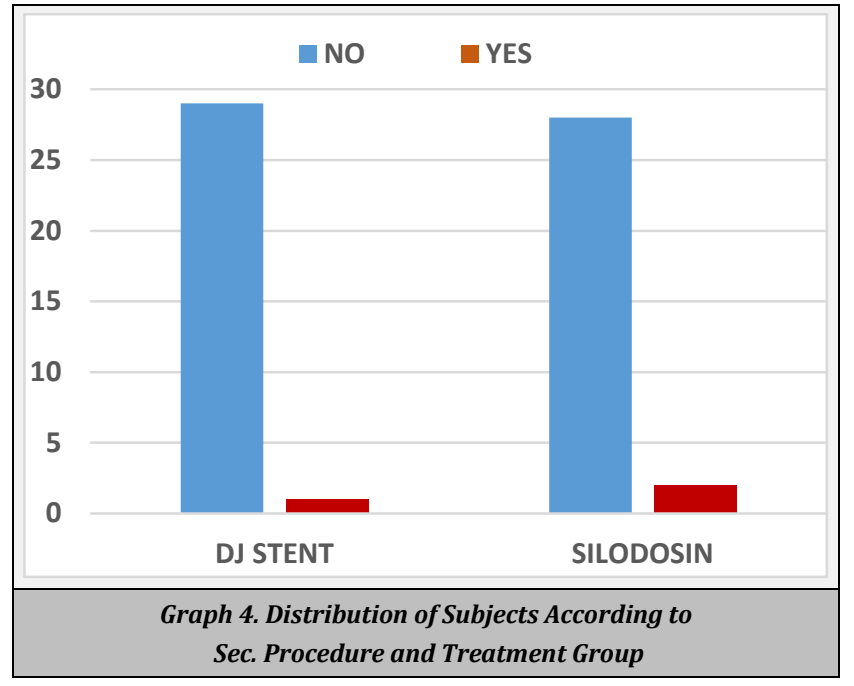

\begin{tabular}{|ccccc|}
\hline & Group & Mean & S.D. & P Value \\
Age & DJ stent & 35.43 yr. & 12.011 & .484 \\
Serum & Silodosin & 37.57 yrs. & 11.467 & \\
Creatinine & DJ stent & 1.113333 & .3159368 & .624 \\
Operation Time & Silodosin & 1.150000 & .2583102 & \\
& DJ stent & 33.23 mins. & 4.599 & $.003 \mathrm{~S}$ \\
& Silodosin & 29.90 mins. & 3.763 & \multirow{2}{*}{ Hospital Stay } \\
& DJ stent & 2.60 days & 1.102 & $.009 \mathrm{~S}$ \\
\hline \multicolumn{5}{|c}{ Table 1. Comparison of Mean Age, Serum Creatinine, } \\
& Silodosin & 1.83 days & 1.085 & \\
& Operation Time, and Hospital Stay \\
\hline \multicolumn{5}{c}{}
\end{tabular}

\begin{tabular}{|cccc|}
\hline \multirow{2}{*}{ Sec. Procedure } & DJ Stent & Silodosin & Total \\
& 29 & 28 & 57 \\
No & $96.7 \%$ & $93.3 \%$ & $95 \%$ \\
& 1 & 2 & 3 \\
Yes & $3.3 \%$ & $6.7 \%$ & $5.0 \%$ \\
\multirow{2}{*}{ Total } & $\mathbf{3 0}$ & $\mathbf{3 0}$ & $\mathbf{6 0}$ \\
& $\mathbf{1 0 0 . 0 \%}$ & $\mathbf{1 0 0 . 0} \%$ & $\mathbf{1 0 0 . 0 \%}$ \\
\hline \multicolumn{4}{|c}{ Table 2. Distribution of Subjects According to } \\
Sec. Procedure and Treatment Groups
\end{tabular}

\section{DISCUSSION}

Advances in intracorporeal lithotripsy suggest that URSL is the treatment modality of choice for ureteric stones.[10] DJ stenting even after an uncomplicated URSL is done in many centres. There are no guidelines for the choice of stents for the treatment of ureteral stones. A survey in 2015 showed that 63 percent of the urologists stented patients after URSL. [11] The most significant drawbacks of ureteral stenting in most patients are the complications due to stent in situ.[12] Pain and frequency are the most common complications caused due to stent. Also, stents irritate and stimulate the bladder mucosa, resulting in dysuria, urgency, haematuria, and infection. DJ stents are known to cause ureteric oedema and inflammation, which may have long term sequelae. Hence it is preferable to use stents for the short term only.[13] Usually, late complications of DJ stent include hydronephrosis, encrustation, migration, and fragmentation during removal.[14] Also, with longer indwelling time, there is a higher incidence of incrustation, infections, secondary stone formation, and obstruction of the stented tract. Patients are stented for 7 to 28 days following URSL, but the ideal duration of stenting is not known.

Byrne RR et al. recommended against routine DJ stenting after uncomplicated URSL. Of 60 patients treated in their study, 38 were stented with a mean duration of 5.2 days. Twenty-two patients were without a stent in their study. There was less flank discomfort group without a stent. Suprapubic pain was more in the patients with stent $(\mathrm{p}=$ 0.002). LUTS was similar between in all patients on day 1 . LUTS was significantly higher in the DJ stent group on day 6 ( $p<0.001$ ). They concluded that DJ stenting following URSL might be avoided if possible.[15]

Hai Wang et al. did a meta-analysis in 2017 regarding with or without stent in the treatment of ureteric calculus. Stone clearance and pain relief are no better with stent compared to the non-stented group. Stenting is associated with far more significant LUT symptoms, infections, and haematuria than the non-stented group.[16]

Silodosin is a highly selective $\alpha-1 \mathrm{~A}$ receptor blocker. Tsuzaka and colleagues, ${ }^{[17]}$ reported that an $\alpha-1 \mathrm{D}$ adrenoceptor blocker was inferior to the $\alpha$-1A-adrenoceptor blocker in medical stone expulsion. The affinity of silodosin for the $\alpha-1 \mathrm{~A}$ subtype was 17 times more than tamsulosin. A systematic review and meta-analysis by Diandong Yang, Jitao Wu et al. regarding the efficacy and safety of silodosin for the treatment of ureteral stones included six RCTs with 916 participants. This showed silodosin to be superior to controls in terms of calculus expulsion in all RCT. Also, the stone expulsion time, and analgesic requirements which were secondary efficacy endpoints, were better with silodosin. They concluded that silodosin has a low side effect and is safe and effective in treatment for ureteric stones.[18] Our study also showed that silodosin is safe and effective and can be used instead of DJ stent in patients with uncomplicated ureteroscopic lithotripsy.

\section{CONCLUSIONS}

Patients who received silodosin after ureteroscopic lithotripsy had a shorter hospital stay and operative time when compared to the DJ Stent group. The rates of passage of fragments after URSL were significantly higher in the silodosin treated group. However, we did not find any significant difference in emergency visits, rehospitalisation rates, and symptoms on postoperative follow-up. The secondary procedure rates between the two treatment groups were also similar. These findings suggest that DJ stent placement following uncomplicated URSL may not be needed in all patients. This reduces patient morbidity and medical costs. However, a multicentric randomised controlled trial is necessary for a larger population to validate and authenticate the study.

\section{REFERENCES}

[1] Pak CY. Kidney stones. Lancet 1998;351(9118):1797801.

[2] Ramello A, Vitale C, Marangella M. Epidemiology of nephrolithiasis. J Nephrol 2000;13(Suppl 3):S45-S50.

[3] Ahmad H, Azim W, Akmal M, et al. Medical expulsive treatment of distal ureteral stone using tamsulosin. J Ayub Med Coll Abbottabad 2015;27(1):48-50. 
[4] Hollingsworth JM, Rogers MAM, Kaufman SR, et al. Medical therapy to facilitate urinary stone passage: a meta-analysis. Lancet 2006;368(9542):1171-9.

[5] Miller OF, Kane CJ. Time to stone passage for observed ureteral calculi: a guide for patient education. J Urol 1999;162(3 Pt 1):688-91.

[6] Preminger GM, Tiselius HG, Assimos DG, et al. 2007 Guideline for the management of ureteral calculi. Eur Urol 2007;52(6):1610-31.

[7] Davenport K, Timoney AG, Keeley FX Jr. Effect of smooth muscle relaxant drugs on proximal human ureteric activity in vivo: a pilot study. Urol Res 2007;35(4):20713.

[8] Nakada SY. Tamsulosin: ureteric motility. BJU Int 2008;101(9):1061-2.

[9] Andersson KE, Forman A. Effects of calcium channel blockers on urinary tract smooth muscle. Acta Pharmacol Toxicol (Copenh) 1986;58(Suppl 2):193-200.

[10] Turk C, Petrik A, Sarica K, et al. EAU Guidelines on interventional treatment for urolithiasis. Eur Urol 2016;69(3):475-82.

[11] Dauw CA Simeon L, Alruwaily AF, et al. Contemporary practice patterns of flexible ureteroscopy for treating renal stones: results of a worldwide survey. J Endourol 2015;29(11):1221-30.
[12] Keeley FX Jr, Timoney AG. Routine stenting after ureteroscopy: think again. Eur Urol 2007;52(3):642-4.

[13] Lumiaho J, Heino A, Pietiläinen $T$, et al. The morphological, in situ effects of a self-reinforced bioabsorbable polylactide (SR-PLA 96) ureteric stent; an experimental study. J Urol 2000;164(4):1360-3.

[14] Damiano R, Oliva A, Esposito C, et al. Early and late complications of double pigtail ureteral stent. Urol Int 2002;69(2):136-40.

[15] Byrne RR, Auge BK, Kourambas J, et al. Routine ureteral stenting is not necessary after ureteroscopy and ureteropyeloscopy: a randomized trial. J Endourol 2002;16(1):9-13.

[16] Wang H, Man L, Li G, et al. Meta-analysis of stenting versus non-stenting for the treatment of ureteral stones. PLoS One 2017;12(1):e0167670.

[17] Tsuzaka Y, Matsushima H, Kaneko T, et al. Naftopidil vs silodosin in medical expulsive therapy for ureteral stones: a randomized controlled study in Japanese male patients. Int J Urol 2011;18(11):792-5.

[18] Yang D, Wu J, Yuan H, et al. The efficacy and safety of silodosin for the treatment of ureteral stones: a systematic review and meta-analysis: BMC Urology 2016;16(1):23. 